\title{
Erratum: Edge states in a two-dimensional honeycomb lattice of massive magnetic skyrmions [Phys. Rev. B 98, 180407(R) (2018)]
}

\author{
Z.-X. Li, C. Wang, Yunshan Cao, and Peng Yan \\ (Received 23 February 2019; published 11 March 2019)
}

DOI: 10.1103/PhysRevB.99.099901

We have found the following errors in the original article. A wrong Dzyakoshinskii-Moriya interaction parameter was adopted for calculating Fig. S2 in the Supplemental Material. Figures S2(b) and S2(c) should be replaced by the revised figure below. The longitudinal and transverse coupling constants should be $I_{\|}=-2.902 \times 10^{-6} \mathrm{~J} / \mathrm{m}^{2}$ instead of $-1.965 \times 10^{-6} \mathrm{~J} / \mathrm{m}^{2}$ and $I_{\perp}=-1.456 \times 10^{-5} \mathrm{~J} / \mathrm{m}^{2}$ instead of $-1.191 \times 10^{-5} \mathrm{~J} / \mathrm{m}^{2}$, respectively.

These changes do not affect the results and conclusions of the paper.
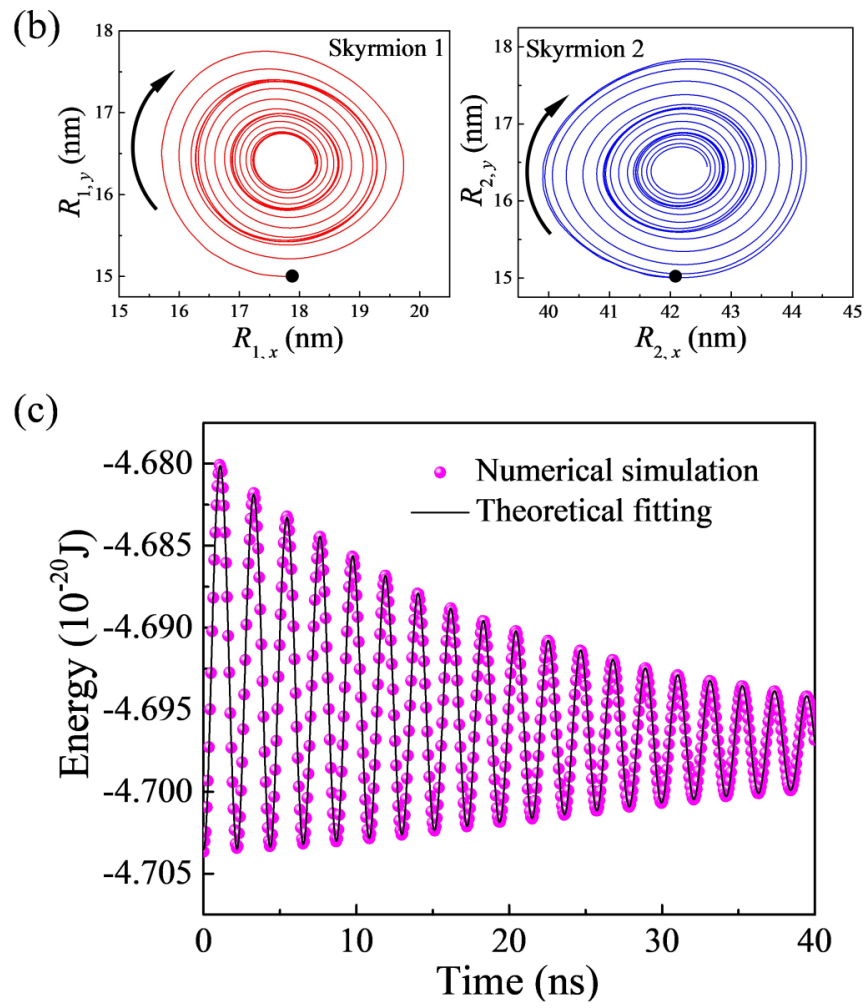

FIG. S2. (b) The trajectories of skyrmion guiding centers. (c) The simulation and fitting results of the system energy. 